\title{
La ausencia de la formación gramatical en la enseñanza del español en México y sus efectos
}

The absence of grammatical learning in the teaching
of Spanish in Mexico and its effects Irma Munguía Zatarain* Universidad Autónoma Metropolitana Iztapalapa, Ciudad de México, México ORCID: http://orcid.org/0000-0003-1873-905X

ISSN: ISSN-OI85-4259; e-ISSN: 2007-9176 DOI: http://dx.doi.org/10.28928/revistaiztapalapa/792015/atcI/munguiazataraini

\section{Resumen}

En este trabajo se analizan las causas del fracaso en el desarrollo de habilidades para leer y escribir con fluidez y corrección en español, entre estudiantes que ingresan al medio universitario; a partir de ello se propone la recuperación de la enseñanza disciplinaria de contenidos gramaticales en el aula; es decir, la necesidad de elaborar una gramática didáctica que motive el desarrollo de una conciencia lingüística en los estudiantes, la cual sería el mejor disparador para que empleen su lengua con mayor control, eficiencia y corrección. Palabras clave: gramática, conciencia lingüística, enseñanza de lengua materna, lingüística, escritura

\section{Abstract}

This work analyzes the causes why students who enter the university environment fail in their ability to read and write fluently and correctly. The analysis serves as a basis for a proposal of the recovery of disciplinary grammar content teaching at school; that is to say, it underlines the need to make a didactic grammar of Spanish that encourages the development of linguistic awareness in students, which would best trigger their using the language with greater control, efficiency and correction.

Key words: Grammar, Linguistic Awareness, Teaching of the Mother Tongue, Linguistics, Writing

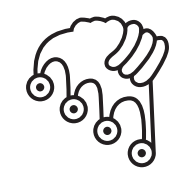

IZTAPALAPA

Agua sobre lajas

Departamento de Filosofía, Área de Problemas Lingüísticos de México irmamunguiaz@hotmail.com 
mediados de los años setenta comenzaron a agudizarse en México los problemas y fracasos de los estudiantes de educación primaria, secundaria y bachillerato, en su desempeño en las materias relacionadas con la enseñanza del español. Muy pronto, los del nivel profesional también empezaron a exhibir una notable deficiencia en el manejo de su lengua. Recientemente, el Consejo Regional del Área Metropolitana (CRAM) de la Asociación Nacional de Universidades e Instituciones de Educación Superior (ANUIEs) impulsó una investigación acerca de las habilidades lingüísticas de los estudiantes de primer ingreso en el medio universitario, ${ }^{\mathrm{I}} \mathrm{y}$ los resultados se publicaron como libro colectivo (González Robles, 2014). En un artículo incluido en ese volumen hago un recorrido crítico de lo que fueron los distintos cambios y reformas, así como un análisis de las fallas que han tenido los planes y programas del Sistema Educativo Nacional; también

I A finales de 2009 el Cram de ANUies conformó un equipo de investigadores, de distintas instituciones de educación superior en el DF (UNAM, UAM, ITAM, UACM), para que diseñara y realizara una investigación acerca de las habilidades lingüísticas que presentan los estudiantes de primer ingreso en las instituciones de educación superior en el área metropolitana de la ciudad de México. El instrumento construido fue el Examen de Habilidades Lingüísticas (Exhaling), y se aplicó a 435I estudiantes; las instituciones que participaron en la evaluación fueron el Centro de Investigación y Docencia Económicas (CIDE), la Escuela Nacional de Antropología e Historia (enAh), el Instituto Nacional de Bellas Artes (INBA), el Instituto Tecnológico Autónomo de México (IтAm), el Tecnológico de Estudios Superiores de Ecatepec (TESE), la Universidad Anáhuac, la Universidad Autónoma Metropolitana (UAM), la Universidad Nacional Autónoma de México (UNAM), la Universidad Iberoamericana (UIA), la Universidad Intercontinental (UIC) y la Universidad Pedagógica Nacional (UPN). El producto de esta investigación, en la que participé, se publicó como libro colectivo (González Robles, 20I4). En este texto, se reportan los resultados que obtuvieron los estudiantes en el ExHALING, en los aspectos de comprensión auditiva, comprensión lectora, producción escrita y conciencia lingüística; se consideraron los resultados por carrera, por institución de origen - tipo de bachillerato- e institución en la que están inscritos los alumnos en el nivel profesional; también se tomaron en cuenta datos personales y sociofamiliares, trayectoria escolar previa, así como su actitud ante el aprendizaje de la lengua. Al final, se incluyeron además algunos comentarios y recomendaciones sobre la enseñanza del español como lengua materna, dirigidos a las autoridades encargadas de dictaminar y promover los cambios en las políticas lingüísticas en México. 
incluyo algunas líneas para elaborar recomendaciones y propuestas de solución (Munguía, 2014:267-283).

En el presente artículo pretendo ampliar el análisis y esbozar una propuesta más completa e integral que ayude a mejorar la formación escolar preuniversitaria en el campo del lenguaje. Mi idea se apoya en la necesidad de enseñar a los alumnos algunos principios de lingüística y de gramática; obviamente, se trata de diseñar una gramática didáctica adecuada, sin terminología especializada excesiva, que propicie una reflexión metalingüística detenida y seria durante la educación preuniversitaria - desde primaria hasta bachillerato-, lo cual propiciaría el desarrollo de una conciencia lingüística. Sin duda, un estudiante con conciencia de su lenguaje y con algunos conocimientos gramaticales estaría en mejores posibilidades de ejercer un mayor control sobre el empleo de su lengua y sobre la corrección de sus escritos. Además, las prácticas escolares de lectura y de redacción contarían con un sustento teórico, lo cual le daría mayor solidez a la formación de los alumnos en esta materia.

Para ello, comienzo por hacer un breve análisis de lo que fue la gramática tradicional en el pasado, la estructural en la reforma de los años setenta, así como el significado de la última modificación, que se caracterizó por incluir el enfoque comunicativo, en los años noventa, e ideas para transformar la enseñanza del español en un ejercicio más exitoso.

\section{La enseñanza tradicional}

Para entrar en materia, creo que vale la pena preguntarse cómo era la enseñanza tradicional del español antes de las reformas educativas de 1972-1973, así como qué resultados se tenían en el desempeño de los alumnos, en lo que se refiere a sus capacidades de leer y escribir bien. Analicemos brevemente las siguientes consideraciones:

a) Es interesante observar que de los años sesenta hacia atrás, por lo general, a un egresado del bachillerato se le consideraba un individuo medianamente culto, que escribía, leía y se expresaba bien; estaba más o menos preparado para enfrentar la educación superior, y en las licenciaturas a las que accedía, no tenía los problemas tan graves que ahora sufren los alumnos para leer y escribir; en su mayoría, los estudiantes tenían éxito en concluir adecuada y decorosamente sus estudios profesionales. ${ }^{2}$ Actualmente, estas dificultades se han convertido en verdaderos

2 Para un análisis amplio de lo que ha sido la educación en México, véase Latapí, 1998. Gutiérrez Legorreta (2009) hace un interesante estudio sobre el caso del bachillerato, y Zorrilla (2004) sobre la educación secundaria. 
obstáculos para que las instituciones de educación superior, por ejemplo, logren la deseada eficiencia terminal. Muchísimos estudiantes se retrasan en concluir sus licenciaturas debido a sus problemas para elaborar una tesis; y durante su formación profesional, se sienten perdidos $-y$ los maestros frustrados-, puesto que son incapaces de redactar resúmenes, reseñas, ensayos y diversos trabajos académicos que suelen solicitarse en el medio universitario. Las múltiples quejas de los profesores en este sentido son ya muy conocidas.

Por supuesto, para analizar con más detalle este asunto, hay que considerar el significativo aumento en la matrícula en las instituciones de educación superior a partir de I960 (Fresán, 1998) y las enormes dificultades que ello entraña.

Si esto es así, habría que ver cómo era la enseñanza del lenguaje en esa época, antes de que se masificara la educación y se hiciera una gran reforma, en la década de los setenta (Guevara Niebla, 2013), para poder dirimir qué aspectos tenía de positivos y qué otros se tendrían que corregir. Es de suma importancia considerar que en la educación tradicional, los programas de español tenían contenidos disciplinarios gramaticales que enseñar en la escuela, aunque estuvieran mal planteados o mal sustentados desde el punto de vista de las teorías lingüísticas modernas. Pero los profesores de español se sabían obligados a enseñar temas gramaticales específicos: el sustantivo, el pronombre, el verbo, sus clases, sus funciones, los tipos de oraciones, etc. Los niños aprendían cómo era la gramática de su lengua, aunque con metodologías antiguas y demás. Pero finalmente, los egresados de la educación preuniversitaria manejaban su lengua mejor que como lo hacen actualmente los alumnos del mismo nivel. Veamos un ejemplo muy sencillo: antes de la reforma educativa de los años setenta, ante una oración como A Julio le gusta el cine francés la mayoría de los alumnos sabía que a es una preposición - palabra invariable que no marca género ni número-; Julio es un nombre propio masculino, singular; le es un pronombre personal de tercera persona singular; gusta es un verbo conjugado en presente de indicativo, tercera persona del singular, etc.; también era capaz de saber que el sujeto es el cine francés, pues es la frase que concuerda con el verbo, y a Julio es un complemento indirecto, que se repite en el pronombre le. Actualmente, muy pocos estudiantes del nivel universitario tienen este conocimiento. ${ }^{3}$

b) Otro aspecto importante que conviene señalar es que antes de la mencionada reforma, la mayor parte de los docentes de las distintas asignaturas realizaban la tarea de enseñar a comentar y construir un texto sin faltas de ortografía y bien estructurado; no era una tarea que solo le tocara al profesor de español. En aquella

3 Véase Munguía, 2014:221-238. 
época no se banalizaban estos aspectos de la escritura. Todos o casi todos los profesores contribuían en esta labor.

c) Sin embargo, debe reconocerse que la enseñanza tradicional era de tipo memorístico y fuertemente normativo, prescriptivo; los profesores de español obligaban a los estudiantes a memorizar paradigmas de verbos, listas de preposiciones y conjunciones, clases de palabras, definiciones de las distintas categorías gramaticales, reglas sintácticas, normas ortográficas, reconocimiento de funciones gramaticales como sujeto, objeto directo, etc. En esa época los profesores y las instituciones no se percataban de que estaban enseñando el español a los niños y adolescentes como si estos no lo conocieran, como si no fueran hablantes de esta lengua. ${ }^{4}$

\section{La reforma educativa de los años setenta y el estructuralismo}

A principios de los años setenta se hizo el intento de corregir las fallas en la enseñanza con una gran reforma educativa, pero creo que, en lo que se refiere al español, se realizó de manera equivocada, por varias razones:

Se partió solamente del desprestigio del que habían sido objeto los estudios gramaticales en los últimos años, debido a las fuertes críticas al estructuralismo y a que se consideró que la gramática y sus normas de corrección no servían para escribir mejor. Se hizo esto sin analizar con detenimiento si esa enseñanza tradicional había realizado algún tipo de aporte en la formación de los jóvenes. Las críticas se basaban en que la gramática tradicional era memorística, prescriptiva, decididamente normativa, además de que sus definiciones estaban mal elaboradas, puesto que mezclaban criterios distintos en sus definiciones (la diacronía con la sincronía, la semántica con la morfología y la sintaxis, entre otros). ${ }^{5}$ Pero el error fue incluir nuevas nociones gramaticales de tipo teórico aportadas por el estructuralismo lingüístico, que tampoco funcionaron; ese conjunto de nociones y de terminología específica que se integró en los programas de estudio, de manera incompleta, aislada, desarticulada,

4 Todavía en la actualidad se comete el mismo error cuando se habla de gramática.

5 Cómo no iba a ser así, si la llamada gramática tradicional estaba inspirada en la obra de Andrés Bello, publicada originalmente en 1847; de Antonio de Nebrija, original de I492; las versiones de 1920 y 1942 de la Real Academia Española; esta última dice: "La Ley de 9 de septiembre de I857, en su artículo 88 declara que la GRAmática de la Academia Española es texto obligatorio y único en las escuelas de enseñanza pública" (RAE, I942:5). Considérense también las gramáticas adaptadas a la enseñanza de Gili Gaya, 196I, Alonso y Ureña, 1969, entre otras. Para una crítica a la gramática tradicional, véase Roca-Pons, 1970:9-29. 
no había sido asimilada adecuadamente por los docentes, cuando estos se vieron obligados a enseñarlas en la escuela sin conocer bien a qué correspondían, a qué sistema teórico pertenecían y de qué podrían servir en la práctica escolar.

Como ya señalé, la reforma se estructuró sobre la base de que la gramática no servía para enseñar ni a leer ni a escribir mejor. Por eso, aparte de los temas teóricos, en los programas nuevos aparecieron otros de orden "práctico" como la descripción, la narración, la crónica, las fichas de trabajo, la lectura de textos diversos y la identificación de ideas principales y secundarias, con la intención de que los alumnos redactaran y leyeran distintos tipos de escritos. Pero estaban también los temas gramaticales, tomados tal cual de la teoría lingüística, sin haber elaborado antes una didáctica específica que hubiera ido presentando gradualmente algunos de esos tópicos de manera adecuada, si es que venía al caso tratarlos en la escuela. De pronto, sin un entrenamiento apropiado y suficiente, los profesores ${ }^{6}$ se vieron obligados a hablar del paradigma, el sintagma, el fonema, el morfema, el significante, etc., todas ellas nociones relacionadas con un modelo teórico lingüístico - desconocido hasta entonces-, que no tenían por qué enseñarse en las aulas de la manera como se hizo, puesto que perdían su sentido. Es muy sabido que un principio teórico perteneciente a un sistema científico que pretenda explicar un fenómeno no puede aislarse ni se le puede buscar una aplicación útil de manera directa e inmediata, sino que adquiere sentido dentro del contexto al que pertenece.

Muchos profesores que no estaban formados en esta corriente lingüística - probablemente la mayoría - no le encontraron significado a las modificaciones de la reforma, pues entendieron que solo se trataba de un cambio en la terminología, y que tal vez únicamente se intentaba hacer parecer a la gramática como algo muy complejo y complicado.

Si la enseñanza del español ya estaba siendo cuestionada, debido a los métodos antiguos de la gramática tradicional, con la reforma de los años setenta terminó por desprestigiarse, pues en el aula la gramática se consideró una materia especialmente aburrida e inútil para los fines prácticos de enseñar a leer y escribir. Piénsese, por ejemplo, cómo no va a ser tedioso estudiar como tema de un día, en la escuela secundaria o en el bachillerato, la diferencia entre paradigma y sintagma, o entre diacronía y sincronía (nociones teóricas de un complejo sistema lingüístico), y luego dejarlo como tema visto para ser evaluado al final del curso. Como dije antes, no se elaboró una gramática didáctica, no se hizo una transposición didáctica de corte estructural,

6 Formados en la gramática tradicional. 
y creo que ese fue el gran error: incluir temas sueltos, sin contexto, sin sentido, con una terminología que muchísimos docentes y alumnos nunca entendieron.

Además, se incluyeron temas que aludían a la enseñanza de la redacción, por ejemplo, la descripción, la narración, la entrevista, el reportaje, etc. Sin embargo, los profesores no tenían elementos didácticos para lograr que los alumnos los produjeran en clase; en los libros de texto de secundaria ${ }^{7}$ vemos que se pide al alumno que describa, por ejemplo, su propio rostro o su casa, o que narre cómo fueron sus vacaciones. Nótese que se trataba, muchas veces, de temas improvisados, que tal vez no interesaran al alumno, como materia de estudio escolar. Habría que considerar que comúnmente, cuando los jóvenes asisten a una institución educativa van con la ilusión de aprender algo novedoso, interesante, algo desconocido o ajeno o hasta difícil; y pedirle que describa a su perro o su gato o que narre qué hizo el día anterior puede resultar totalmente ocioso, inadecuado y aun frustrante o desconcertante. En el caso de la lectura, los profesores - y los libros de texto- buscaron materiales de las otras asignaturas, de cultura general, de divulgación de la ciencia o de curiosidades, para poder motivar a los estudiantes a leer y luego encontrar ideas principales y secundarias, entre otros elementos. Pero piénsese en el poco interés que representaban los contenidos de esos textos para los alumnos - y para los mismos maestros-, porque no se trataba de leer un texto para aprender algo, como se suele leer un artículo para el curso de historia o de biología. En el caso de las materias de español se leía para localizar ideas principales y secundarias que luego no servirían para otra actividad, pues no eran temas o contenidos que hubiera que estudiar $y$ aprender. Se trataba solo de ejercitar o de conocer estrategias para mejorar su habilidad lectora; pero vemos que en 40 años de seguir este tipo de prácticas estamos muy lejos de lograr que los alumnos lean y escriban bien.

Además, considérese que los profesores de español no estaban entrenados específicamente para enseñar a leer y escribir, como tampoco lo está ningún maestro de las demás asignaturas; pero, finalmente, cada profesional ha tenido que enfrentarse a la tarea de leer libros y artículos especializados, hacer tesis, tesinas y diversos trabajos académicos, con buena redacción y ortografía, con un adecuado manejo del aparato crítico, de tal manera que los más autorizados para comentar las ideas principales de una lectura o de opinar si está bien argumentado un escrito, son los especialistas en esa disciplina, y no los profesores de español; la mayoría de estos últimos han sido formados en licenciaturas - o escuelas normales- del área de

7 Véanse los libros de texto de secundaria aprobados por la Secretaría de Educación Pública y distribuidos después de 1973 . 
lengua y literatura, ${ }^{8}$ y que por lo tanto, lo que saben o deben saber es gramática, lingüística y literatura. Curiosamente, en nuestro medio siempre se ha asumido que quienes deben saber enseñar a leer $y$ a escribir son los maestros que estudiaron lingüística, gramática y literatura, pero al mismo tiempo se ha repetido de manera insistente que la gramática no sirve para saber redactar. Es una contradicción que nunca se ha examinado con cuidado y detenimiento. El caso es que los profesores de español asumieron la tarea de enseñar redacción y lectura, se improvisaron como los expertos en el desarrollo de esas habilidades, y han realizado muchos de ellos una labor muy loable, sin duda, pero con resultados insatisfactorios a nivel nacional.

\section{La reforma de 1993}

Debido al fracaso de la reforma educativa de la década de los setenta, en 1993 se realizó una nueva reforma. Como se sabe, se implantó el enfoque comunicativo funcional que, sin duda, tenía las mejores intenciones de lograr un cambio significativo; dice Rebeca Barriga: "Definitivamente, el enfoque comunicativo es, en sí mismo, una propuesta excelente; está en consonancia armónica con la historia natural del hombre (Wittgestein, 1986:15) y su naturaleza intrínsecamente discursiva. En definitiva también los ltge [libros de texto gratuitos de español] ofrecen caminos para fortalecerla" (2012:100).

En lo que se refiere a la materia de español, esta nueva reforma trató de enseñar "haciendo", es decir, los alumnos aprenderían a escribir "escribiendo", a leer "leyendo" textos cuyas temáticas fueran cercanas a sus intereses y motivaciones. Entonces, se prescindió de toda teorización, por ello se eliminaron casi todos los temas relacionados con gramática, y solo quedaron algunos aislados y atomizados, de tal suerte que en los programas de estudio no existe realmente un contenido explícito disciplinario que el alumno tenga que estudiar.

Pero ya transcurrieron más de 20 años y los resultados siguen siendo muy desalentadores (Moreno de Alba, 2009:7-IO; González Robles, 2014). Las diferentes evaluaciones aplicadas ${ }^{9}$ muestran que el enfoque comunicativo está lejos de haber logrado sus fines (Barriga, 20I2:100). Sin duda, creo que la enseñanza de la lengua materna mayoritaria en este país, el español, se ha estado realizando de manera

8 Hasta la fecha sigue ocurriendo así.

9 Por ejemplo, los Exámenes de Calidad y Logro Educativos (excale) y la Evaluación Nacional de Logro Académico en Centros Escolares (Enlace), entre otros. 
equivocada. Examinemos con cierto detalle algunos aspectos de este enfoque comunicativo:

a) Se tomó como dogma la idea de que conviene desterrar a la gramática de la enseñanza, puesto que no sirve para mejorar el empleo de la lengua. Entonces, los contenidos de esta rama de la lingüística en los programas de educación son mínimos, insignificantes, están prácticamente proscritos; con esta nueva reforma se recomienda enseñar - las pocas nociones aisladas que quedan - de manera "oculta", no explícita; es decir, los libros de texto ${ }^{\text {io }}$ no hablan de definiciones, de clasificaciones ni de ninguna terminología gramatical, se pretende que los alumnos deduzcan una información teórica a partir de un ejercicio, tarea que difícilmente se puede lograr, pues ningún elemento es aludido por su nombre - como sujeto agente o paciente, verbo transitivo, voz pasiva refleja." Además, el rechazo a la prescripción gramatical y al prurito mal entendido de querer respetar todas las variedades del español, llevó a prescindir de las expresiones "es correcto" o "es incorrecto" en relación con algún uso; se ha optado, en cambio, por expresar que "es adecuado" o " es inadecuado"; ello debido a que existe la convicción de que no se debe enseñar normativamente el lenguaje - cuando sabemos que la mayoría de los hablantes buscan de manera afanosa que se les diga si tal expresión o palabra es correcta o no y, además, sabemos que la escuela es la institución formal encargada de enseñar la variedad estándar de una lengua. Así, algunos de los temas que "se ven" son el sustantivo, el pronombre, el sujeto oracional, pero no se desarrollan, no se estudian, no son temas teóricos sobre los cuales el alumno deba reflexionar y aprender, se revisan como algo accidental que se desprende después de la lectura de un texto que, por cierto, tampoco debe estudiar el alumno, puesto que es un material ocasional que se emplea para que este encuentre ideas principales o para que identifique un personaje en un cuento de la narrativa mexicana, o con cualquier otro fin. Piénsese qué tan inútiles pueden resultar los cursos de español, si en ellos se "trabaja" con este tipo de actividades ocasionales. $\mathrm{Al}$ alumno no le queda claro qué es lo que tiene que aprender.

b) En los cursos de lengua, desde la primaria hasta el bachillerato, se trata de enseñar a describir, a narrar, a argumentar, pero al mismo tiempo no suelen darse los elementos para lograrlo; al respecto, Peón y Rojas-Drummond dicen (20I2:135136) que los libros de texto dan por hecho que los estudiantes saben lo que es un

Io Véanse los libros de texto de educación secundaria autorizados por la SEP, y publicados por distintas editoriales después de 1993. Véase también Zamudio, 20II.

II Véase el interesante análisis que hace Parra (2012:169-2I2) sobre los errores en la forma de enseñar oraciones negativas a niños de primaria. 
argumento - desde el quinto año de primaria-, y les ofrecen ejercicios en los cuales deben identificar los argumentos que expone un personaje en un texto, $y$ de inmediato les piden que preparen sus propios argumentos para participar en un debate; pero nunca dicen qué debe entenderse por argumento. Curiosamente, desde este nivel educativo, el alumno debe escribir artículos de fondo o un artículo editorial, pero sin información sobre cómo se construye una argumentación y sin entrenamiento previo.

c) Se asume el lenguaje solamente como un medio eficaz de comunicación, un instrumento que se puede pulir sin reflexionar acerca de lo que es y cómo funciona, cómo se usa; se busca afanosa, pero infructuosamente, la forma de que los jóvenes desarrollen la habilidad de emplearlo bien, pero no se considera importante que tomen conciencia de lo que son los sistemas lingüísticos, cómo están insertos en una cultura, cómo varían dependiendo de factores geográficos, sociales e históricos. En la escuela, el lenguaje es visto únicamente como una herramienta que sirve para escribir y leer acerca de otras disciplinas; es entonces una materia de apoyo, un taller a donde se asiste con el fin de familiarizarse con técnicas o estrategias para volverse hábil en la escritura, la lectura y, en ocasiones, en la investigación documental. El español perdió sus contenidos disciplinarios y, tácitamente, su estatus como materia formativa importante y seria; y con ello comenzó a fracasar en su propósito de enseñar a escribir y leer mejor.

d) No se reconoce la utilidad, el interés o la necesidad de estudiar el lenguaje por sí mismo, se considera una actividad ociosa, inútil y aburrida; desde esa perspectiva no se logra mirarlo como el reflejo de una estructura cognoscitiva muy particular, cuyo estudio arrojaría mucha luz sobre cómo funciona el cerebro humano y cómo, en esa medida, su estudio sería tan interesante como lo es el de la biología o de la economía. Tampoco se examina que el lenguaje no solo es el método más eficaz de comunicación, sino que sirve para múltiples tareas, por ejemplo para construir ciencia, para sistematizar y ordenar ideas, para simular una comunicación inexistente o imaginaria — función que ningún lenguaje animal realiza—, para hablar de mundos posibles o simplemente de hechos pasados o futuros - que tampoco ningún otro sistema de comunicación animal o de otra clase realiza-, para mentir y para prometer, para construir metáforas que aludan a realidades no obvias, para expresar ironía, para parodiar, para crear significados nuevos y estéticamente placenteros; por supuesto, también sirve para jugar, como puede verse en el caso de muchos chistes y acertijos lingüísticos. El lenguaje no es solo comunicación. Se olvida que los humanos no "inventamos" las lenguas para comunicarnos, sino que nos comunicamos porque poseemos una facultad del lenguaje particularmente 
distinta de la que tienen otras especies de animales; es una facultad muy vinculada con la inteligencia humana, además de que las lenguas son parte de la identidad de los pueblos y están estrechamente asociadas con la cultura; no en balde han sido objeto de estudio desde la antigüedad. ${ }^{12}$

e) En el medio educativo en México, la enseñanza del español está orientada solamente al desarrollo de habilidades para entender y producir mensajes correctos, fluidos y adecuados, pero se hace prescindiendo de reflexiones teóricas y del estudio de su sistema gramatical. Habría que pensar si es posible tener éxito en el desarrollo de habilidades - de corte intelectual, como la lectura y la escritura - sin ninguna teoría, sin explicaciones convincentes gramaticalmente sustentadas o sin hablar de principios de corrección o de variación lingüística. El español se concibe como un instrumento, un artefacto que usa el hablante para comunicarse, y por ello está al servicio de las demás asignaturas, aquellas que sí tienen contenidos disciplinarios que enseñar, como la de química que establece - entre muchos temas más- que el agua está formada por dos partículas de hidrógeno y una de oxígeno; o el álgebra, que el binomio cuadrado perfecto tiene tales propiedades, sin cuestionar si esos conocimientos o aprendizajes le sirven al alumno de manera directa e inmediata en algo que tenga que ver con su entorno — como se le ha exigido a la gramática-; se entiende que son conocimientos sobre ciencia, cultura general y sobre el mundo que el alumno debe conocer y que le serán útiles después, de alguna manera. $\mathrm{Y}$ es indiscutible que esos conocimientos ayudan a lograr la formación de los estudiantes, a la conformación de su cultura general, a su iniciación en la ciencia y en la forma de observar y explicar el mundo. Pero no se piensa que este objetivo se lograría de una manera más integral, más completa, si también aprendieran nociones gramaticales que tanto ayudan en el establecimiento de relaciones lógicas, entre otras funciones más.

Sin duda, el estudio de la gramática no determina el éxito en el desarrollo de habilidades prácticas en el uso del lenguaje, pero sí puede contribuir fuertemente en el desarrollo del pensamiento abstracto. Mucho se ha hablado de que el estudio de las lenguas naturales y los lenguajes formales, como la lógica y las matemáticas, son centrales en el proceso de formación de los estudiantes (Di Tullio, 20IO:I5), y creo que no debiera seguir desdeñándose esa oportunidad.

f) Así pues, en este nuevo enfoque la comunicación ocupa un espacio central; por ello se procura que el niño o el adolescente hable, se exprese y se comunique con sus compañeros, que logre sostener un debate, una discusión en clase; pero todos sabemos que los jóvenes se comunican entre ellos perfectamente mediante el lenguaje

12 Para un comentario sobre este punto, véase Di Tullio, 2010:13-18. 
oral y el escrito; son más capaces que muchos adultos de dirigirse entre ellos mensajes de distinta índole: en recados escritos en papelitos, en mensajes de texto por teléfonos celulares, por correo electrónico, Twitter, Facebook, etc. — esto último, sobre todo, en el medio urbano. Desde el nivel preescolar hasta el de educación superior, los estudiantes conocen y emplean eficazmente su lengua para comunicarse entre ellos y con el resto de su entorno. No es necesario fomentar más esto en las aulas, sino repensar en qué contenidos disciplinarios relacionados con la gramática y la lingüística pudieran ayudarles a desarrollar una conciencia lingüística que, a su vez, podría ser fundamental en ese desarrollo de habilidades para escribir y leer mejor; es la conciencia gramatical y lingüística la que permitiría a los estudiantes saber por qué son correctos ciertos usos y por qué se consideran incorrectos otros, es la conciencia la que les permitiría valorar las distintas variedades del español, así como considerar la importancia que tienen las lenguas mexicanas, entre otras cosas más.

g) Existe en la educación preuniversitaria — primaria, secundaria y bachillerato- cierto "paternalismo", cierta tendencia por hacer las clases de español fáciles, útiles, accesibles y divertidas, un afán por que los alumnos adviertan que se trata de una asignatura no aburrida - como se ha considerado la gramática- que les servirá para otras materias; en esas otras materias, el asunto es serio, ahí sí tienen que aprender, estudiar, reflexionar y hasta sufrir. En la clase de español no, solo es para comunicarse, para hablar, para escribir sobre el tema del día, ya sea sobre las propiedades de las lechugas o del limón, de las mascotas, de la ecología, de lo que propongan los libros de texto o de lo que improvisen los presionados maestros de español. La enseñanza del español se realiza con la preocupación central de que el alumno hable o escriba sobre asuntos que le interesen, que lo estimulen, es decir, se trata de entretener, motivar o divertir a los estudiantes, como si la educación fuera un asunto de pasarla bien, de jugar y regodearse en los asuntos e intereses de los estudiantes. Como mencioné antes, se olvida que tal vez las expectativas de estos sean enfrentarse a contenidos difíciles pero interesantes y que, en general, estén dispuestos a aprenderlos, a estudiarlos. ${ }^{13}$

En los cursos de español o de lengua ha habido un interés sobrevalorado de enseñar contenidos prácticos, de dar al alumno instrumentos que le sirvan de manera clara y directa en algo útil, inmediato. Ha habido un olvido o un desplazamiento sistemático de la importancia de la reflexión teórica —en este caso, metalingüística- y de que esa reflexión es formativa en el sentido más amplio de la palabra, como ya ha quedado repetidamente dicho.

I3 Para un comentario sobre estas ideas, véase Peón y Rojas-Drummond, 2012:138-I39. 
En suma, ambas reformas educativas creyeron inútil el estudio de la gramática y lo eliminaron paulatinamente de los currículos de todo el sistema educativo, y ese estudio se sustituyó con talleres o cursos que se supone promoverían la comunicación, la redacción y la lectura. Así pues, desde hace aproximadamente 40 años ningún joven realiza una reflexión sobre lo que es su lengua materna, a pesar de que la investigación lingüística ha avanzado de manera impresionante en dar explicaciones sobre su importancia en el conocimiento de lo que somos los seres humanos, su papel en el aprendizaje de segundas lenguas, el problema de las patologías lingüísticas, la importancia de las lenguas autóctonas en la cultura, entre muchas explicaciones más. Y el fracaso escolar a nivel nacional es más que elocuente. ${ }^{14}$ Es urgente buscar una solución drástica y radical a este problema.

Es extraño ver todo lo ocurrido en este terreno de la educación en el país sabiendo que los estudios gramaticales, y su presencia en la formación de los jóvenes, se remontan al siglo I a. de C..$^{15}$ En la Edad Media eran fundamentales en la educación, lo mismo que en los siglos subsiguientes. El estudio de la gramática ha sido central en la conformación de las culturas, no solo de Occidente. Tal vez ya sea hora de pensar si su eliminación de los planes y programas de estudio habrá sido un grave desatino, partiendo siempre de la idea de que si bien el conocimiento gramatical no es una condición suficiente, sí es indispensable para escribir bien.

\section{Qué hacer}

Me parece que el gran problema ha sido, desde mediados del siglo $\mathrm{xx}$, no saber de qué manera enseñar el español como lengua materna, no darse cuenta de la necesidad de haber elaborado una gramática didáctica, no teórica ni descriptiva ni especializada. Invito al lector a pensar en que tal vez cualquier clase de gramática didáctica, ya sea en el marco estructural o generativista, pero didáctica, habría sido útil para que los estudiantes, a lo largo de I2 años de instrucción preuniversitaria, hubieran podido hacer algún tipo de reflexión, por lo menos, sobre lo que son las

14

I5

La primera gramática del griego - Arte gramatical - se le atribuye a Dionisio de Tracia (año Ioo a de C., aproximadamente); fue la base de las posteriores gramáticas del griego, del latín y de varias lenguas europeas. Es interesante señalar que desde esa época las gramáticas eran didácticas y normativas; además, se ocupaban más de la lengua escrita que de la oral, y esto fue así durante dos milenios, puesto que no es sino hasta en siglo $\mathrm{xx}$ - con Saussure y la corriente estructuralista- cuando vuelve a prestarse atención al lenguaje oral. 
lenguas naturales, y en esa medida hubieran logrado hacer un uso más consciente, más razonado y más cuidado de su lengua en el momento de escribir.

Creo que no se ha sabido aprovechar ese conocimiento que los alumnos ya tienen cuando llegan a la escuela y, probablemente, a los responsables de elaborar planes, programas y libros de texto les resultaba raro o extraño "enseñar ese mismo conocimiento" - como sí se hacía antiguamente- y se optó solo por desarrollar habilidades de lectura y escritura, pero prescindiendo de un respaldo teórico lingüístico y gramatical.

Otro elemento que habría que reconsiderar con seriedad, a la luz de las nuevas teorías lingüísticas, es el valor y el sentido de la normatividad, como elemento regulador propio del sistema de la lengua. "La normatividad retarda, controla u orienta el cambio, pero no es una intervención espuria sobre la lengua, sino parte de su propia constitución" (Lara, 2004:46); esa condición tiene que ver con lo que son, socialmente hablando, las variedades estándares y no estándares de una lengua, el estatus de los distintos dialectos sociales y geográficos, así como el papel que deben cumplir las academias, los diccionarios, los profesores y las autoridades en esta materia. Como es muy sabido, el estructuralismo de los años setenta señaló el error que había cometido la gramática tradicional al tratar de enseñar a hablar y a escribir con corrección, a partir de ejercicios de repetición y memorización de reglas, normas y clasificaciones mal elaboradas. Sin duda, en la producción del discurso de una persona intervienen las normas de corrección gramatical, pero también otras de corrección social, de aceptabilidad social, que convendría explicitarlas, conocerlas para usarlas de manera consciente. Se olvida, pues, que la normatividad no es algo ajeno impuesto arbitrariamente desde fuera, sino que forma parte del propio sistema de la lengua, es una fuerza reguladora que actúa dentro del mismo mecanismo de la lengua.

Cabe aquí la siguiente reflexión: aunque los niños desde los dos o tres años de edad ya hablen, no basta con que "hablen", se requiere que en la escuela "aprendan a hablar", "sepan hablar", es decir, sepan emplear su lengua en situaciones concretas de manera adecuada ( $c f$. Lara, 2004:9 ss.). Como se sabe, la particularidad de hablar es algo biológico en el ser humano, pero la capacidad de saber hacerlo de manera eficaz y adecuada es algo que atañe a la esfera social y que la escuela debería proporcionar.

Insisto en la necesidad de reconocer que la educación tradicional no tuvo tan malos resultados, aunque se enseñara gramática preceptiva, prosodia y ortografía. ${ }^{16}$

16 Con ello no quiero dar a entender que la solución se encuentre en el pasado, en la gramática tradicional, sino al contrario, que debe acudirse a las nuevas propuestas, a los grandes avances que ha habido en la investigación lingüística contemporánea. 
Es muy sabido que las gramáticas tradicionales, por ejemplo la de la Real Academia Española (RAE), se elaboraban con una intención abiertamente didáctica, pero piénsese que se estudiaba analogía (morfología), sintaxis, prosodia y ortografía $(1942,7)$ con convicción y de manera sistemática e insistente, y los resultados no eran tan malos en el campo educativo. Ahora no enseñamos nada de gramática, no teorizamos, no torturamos a los alumnos con nociones gramaticales, solo les pedimos que lean y escriban textos diversos, pero sin ningún éxito - esto ya desde hace más de 40 años.

La tarea de la escuela debiera ser estimular la reflexión metalingüística, crear una conciencia lingüística en los estudiantes que les permitiera ejercer cierto control o "monitoreo" de sus expresiones para que empleen su lengua con corrección, con adecuación y con mayores probabilidades de encontrar diversas opciones expresivas y creativas que les ofrece el propio sistema del español; todo ello a partir de la revisión de temas teóricos de tipo gramatical, aparte de posibles cursos prácticos de lectura y redacción que contribuirían al mismo fin, sin mencionar los indispensables cursos de literatura que también debieran ser reconsiderados bajo una nueva óptica.

Es importante tomar en cuenta que la lengua materna no puede enseñarse de la misma manera como se enseña el resto de las disciplinas, dado que los estudiantes ya la conocen y emplean; las lenguas maternas no se aprenden, se adquieren a una edad tan temprana que casi ningún otro conocimiento se puede aprender en esa etapa de la vida (Chomsky, 1989) y además se realiza de una manera distinta, pues no se necesita entrenamiento ni refuerzo para lograrlo; el éxito lo obtienen todos los seres humanos, independientemente de la lengua de su entorno - chino, español, inglés, zapoteco-, incluso a pesar de ciertas situaciones adversas particulares. La educación formal tendría que buscar la manera de que los estudiantes hicieran consciente ese conocimiento inconsciente que tienen y que les permite comunicarse, como de hecho lo hacen. Incluso, para explicar la naturaleza del conocimiento lingüístico ha sido necesaria la construcción de una teoría lingüística, y también sería necesaria la creación de una didáctica particular para enseñar la lengua materna en el medio escolar.

Resulta impresionante y frustrante ver cómo el sistema educativo de este país no ha aprovechado convenientemente que cuando los niños llegan al primer año de educación básica no solo interpretan y producen oraciones que nunca han oído, sino que son capaces de entender, por ejemplo, que ciertas frases son ambiguas, como David encontró a Juana estudiando en la biblioteca, donde se puede leer que el que estudiaba era David o era Juana, y nadie les ha informado sobre problemas de ambigüedad en su lengua; también son capaces de saber que la oración El ingeniero 
construyó ese puente es equivalente a oraciones como Ese puente fue construido por el ingeniero o Lo que construyó el ingeniero fue ese puente, entre otras. También saben que una secuencia como "purro" es una palabra posible en español, aunque todos sabemos que no existe en esta lengua; en cambio "scrut" es una palabra imposible e inexistente, dado que la secuencia $s c$ - en posición inicial de palabra no es propia de nuestro sistema fonético ${ }^{17}$ Este conocimiento inconsciente que tienen todos los

17 Aquí ofrezco solo estos dos ejemplos que están vinculados con conocimiento inconsciente sobre patrones fonéticos, pero se pueden citar otros casos relativos a conocimiento sintáctico, semántico, morfológico, etc. Nada más a guisa de ejemplo, piénsese que ante dudas sobre cuál es la forma correcta (si la oración en el inciso $a$ o en el $b$ ), y cuáles son las razones para explicar su corrección:

a) Se incluyó en la lista a los discapacitados

b) Se incluyeron en la lista a los discapacitados

Si los estudiantes supieran que el sujeto sintáctico en español es la frase que concuerda en número con el verbo, se percatarían de que el enunciado en b) está mal construido, puesto que se está haciendo concordar el verbo con el objeto directo "a los discapacitados". Sabría también que los objetos directos animados en español se introducen con la preposición "a", y que un sintagma prepositivo jamás puede funcionar como sujeto en esta lengua.

Para escribir trabajos académicos suele recomendarse el estilo "impersonal", es decir, preferir las formas ejemplificadas en $a, b$ y $c$, pero no la forma personal como aparece en $d$ :

a) Se estudia bien en esa biblioteca / Se invitó a los estudiantes extranjeros

b) En este trabajo se muestran algunos resultados

c) Los libros de poesía se venden muy bien

d) Estudio o estudiamos muy bien en esa biblioteca / Invitamos a los estudiantes extranjeros

Pero nunca se instruye a los estudiantes sobre qué clase de construcciones tenemos en los incisos anteriores. Sería más interesante que emplearan estas formas sabiendo que solo en a) aparecen oraciones impersonales, puesto que no hay sujeto, es decir, una frase que concuerde con el verbo; en cambio en $b$ ) la oración no es impersonal, se trata de la voz pasiva refleja o pronominal, cuyo empleo es mucho más productivo que la voz pasiva perifrástica en español, y resulta muy útil para producir el llamado "estilo impersonal", dado que el agente no se expresa en estas construcciones; y en c) tenemos la voz media, que es una variante de la voz pasiva. Los estudiantes sabrían que la voz pasiva refleja - y la voz media - tienen un sujeto paciente que es el que concuerda con el verbo. En cambio, en d) el sujeto es tácito o morfológico, está elidido, pero sabemos que es "yo" y "nosotros", respectivamente.

Saber identificar el sujeto y el predicado no es un conocimiento inútil pues, entre otras cosas, permite darse cuenta de que no siempre se escucha mejor el sujeto en posición inicial - como suelen recomendar muchos cursos de redacción-, ello depende de la clase de verbo o de construcción: los verbos inacusativos y la voz pasiva refleja suelen posponer sus sujetos, dado que estos no son agentes, sino pacientes: Se enfermaron los niños, Llegaron los invitados, Se venden naranjas; a diferencia de los sujetos agentivos que preceden al verbo: El profesor entregó los exámenes, El gato cazó una mariposa. 
hablantes de español se debe a que los niños logran identificar, sin entrenamiento, sin memorización, que la primera secuencia es posible en su lengua, mientras que la segunda no lo es. Es muy interesante preguntarse cómo un niño llega a saber tanto de su lengua sin una educación escolar o especial, en una etapa tan temprana de la vida — entre dos y cinco años de edad-. Como ya es muy sabido, la respuesta es porque la manera como los humanos adquirimos la lengua materna es muy distinta respecto de cómo aprendemos otro tipo de conocimiento (Chomsky, 1989).

Sin duda, se ha desperdiciado la circunstancia de que los seres humanos, antes de los 20 años — antes de llegar al nivel universitario - tienen una capacidad genética de aprender segundas lenguas con cierta facilidad y, seguramente, de entender con mayor acierto los intrincados mecanismos gramaticales que sustentan y explican su propia lengua materna y, tal vez, emplearlos con más fluidez y dominio, para usar de manera más oportuna y apropiada el español y para aprender segundas lenguas.

Entonces, se trata de construir una gramática didáctica moderna - como ya lo he repetido antes-, con base en los hallazgos que la investigación lingüística ha generado en los últimos 70 años, aproximadamente. Una gramática de esta naturaleza promovería una reflexión metalingüística que, a su vez, daría lugar a una conciencia lingüística en los alumnos; con ello se lograría por lo menos un uso del lenguaje más consciente, más reflexivo, más certero.

Se ha repetido incesantemente que la gramática no sirve para escribir bien, y tampoco para leer; la gramática no sirve para nada práctico. Por ello se eliminó del sistema de enseñanza básica y media superior en este país. Me gustaría terminar este artículo citando a Platón, quien dijo lo siguiente como respuesta a alguien que le preguntó para qué servían las matemáticas: "Dirigiéndose a uno de sus esclavos, le instruyó: 'Dale unas monedas a este hombre que quiere beneficiarse de las mate-

Además, reconocer que no todos los sujetos aluden al agente — como sí ocurre en enunciados del tipo Los albañiles construyeron una cisterna-, sino que el sujeto también puede ser paciente, como en Se construyó una cisterna - en cuyo caso, se trata de la voz pasiva refleja-; en otros casos, el

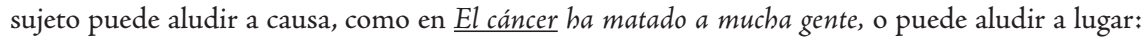
La biblioteca se quedó con el libro, etc. Conocer las clases de oraciones y de verbos puede ayudar a escribir mejor o, por lo menos, a hacerlo con mayor conciencia.

En morfología se podrían estudiar los distintos usos que tienen, por ejemplo, los diminutivos; no siempre se emplean para indicar que una entidad es pequeña o para expresar cariño, como en Una escuelita de barrio, Retrató a un elefantito. También se usan para indicar desprecio como en Nos recibió un hombrecillo; para atenuar, disminuir los efectos de algo como Le haremos una cortadita — dicho por un médico que va a realizar una enorme cirugía-; para expresar ironía como Se compró una casita, cuando se alude a una casa enorme y elegante; incluso, hay formas lexicalizadas como El carrito del supermercado, entre otros usos. 
máticas'” (Martínez, 20I4:16). Tal vez lo mismo hubiera dicho Platón, de habérsele preguntado para qué servía la gramática.

\section{Bibliografía}

Alonso, Amado y Pedro Henríquez Ureña (1969), Gramática castellana, Primer y Segundo Curso, Losada, Buenos Aires.

Baez Pinal, Gloria Estela (2007), La enseñanza del español. Problemas y perspectivas en el nuevo milenio, Universidad Nacional Autónoma de México, México.

Barriga, Rebeca, ed. (20I2), Mitos y realidades del desarrollo lingüistico en la escuela, El Colegio de México, México.

Chomsky, Noam (1989), El conocimiento del lenguaje. Su naturaleza, origen y uso, Alianza, Madrid.

Di Tullio, Ángela (2010), Manual de gramática del español, Waldhuter, Buenos Aires.

Fresán Gómez, Magdalena (ed.) (1998), Tres décadas de politicas del Estado en la educación superior, Asociación Nacional de Universidades e Instituciones de Educación Superior, México.

Gili Gaya, Samuel (196I), Curso superior de sintaxis española, Vox Biblograf, Barcelona.

González Robles y Rosa Obdulia (coord.) (2014), Habilidades lingüisticas de los estudiantes de primer ingreso a las Instituciones de Educación Superior, ANuies, México.

Guevara Niebla, Gilberto (2013), “La disputa por la educación”, en La reforma educativa y otras voces, Educ@upn.mx Recuperado de: <http://www. nexos.com.mx/?P=leerarticulo $\&$ Article $=2103120>$ [I de agosto, 20I4] .

Gutiérrez Legorreta, Lourdes Araceli (2009),"El devenir de la educación media superior. El caso del Estado de México", en Tiempo de educar, año ıo, segunda época, núm. 19, enero-junio, <http://www.redalyc./org/articulo.oa?id=31113164007> [ 5 de marzo, 20I4]

Lara, Luis Fernando (2004), Lengua bistórica y normatividad, El Colegio de México, México.

Latapí, Pedro, (coord.) (1998), Un siglo de educación en México, tomos I y II, Consejo Nacional de la Cultura y las Artes/Fondo de Cultura Económica, México. 
Martínez Morales, Manuel (2014), "Matemáticas, redes y creencias", en La Jornada Semanal, núm. IoI3, 3 de agosto, México.

Moreno de Alba, José G. (2009), Historia y presente de la enseñanza del español en México, unam, México.

Munguía Zatarain, Irma (20I4), “Conciencia lingüística” y"Comentarios y recomendaciones sobre la enseñanza del español como lengua materna en el Sistema Educativo Nacional", en Rosa Obdulia González R. (coord.), Habilidades lingüísticas de los estudiantes de primer ingreso a las Instituciones de Educación Superior, ANuies, México, pp. 22I-238 y 267-283.

Parra Velasco, María Luisa (2012),"Del mito sintáctico a la realidad significativa. Reflexiones sobre las "oraciones negativas" en niños en edades escolares", en Barriga, ed. 20I2: 169-2I2.

Peón, Margarita y Sylvia Rojas-Drummond (2012), "La promoción de habilidades argumentativas en niños de primaria”, en Rebeca Barriga (ed.) (2012), Mitos y realidades del desarrollo lingüistico en la escuela, El Colegio de México, México, pp. 135-168.

Real Academia Española (1942), Gramática de la lengua española. Ediciones Culturales del diario "Novedades", México.

Roca-Pons, J. (1970), Introducción a la gramática, Teide, Barcelona.

Zamudio Mesa, Celia (20II), La revisión de textos en el aula. Una guía para el maestro, Instituto Nacional para la Evaluación de la Educación, México, (Col. Materiales para apoyar la práctica educativa).

Zorrilla, Margarita (2004), "La educación secundaria en México: al filo de su reforma”, en Revista Electrónica Iberoamericana sobre Calidad, Eficacia y Cambio en Educación, México, <http://www.ice/deusto.es/rinace/ vol2ni/zorrilla.pdf $>$ [6 de marzo, 20I4] . 